\title{
KNOTS ARE DETERMINED BY THEIR COMPLEMENTS
}

\author{
C. MCA. GORDON AND J. LUECKE
}

Two (smooth or PL) knots $K, K^{\prime}$ in $S^{3}$ are equivalent if there exists a homeomorphism $h: S^{3} \rightarrow S^{3}$ such that $h(K)=K^{\prime}$. This implies that their complements $S^{3}-K$ and $S^{3}-K^{\prime}$ are homeomorphic. Here we announce the converse implication.

THEOREM 1. If two knots have homeomorphic complements then they are equivalent.

This answers a question apparently first raised by Tietze [T, p. 83].

It was previously known that there were at most two knots with a given complement [CGLS, Corollary 3].

Whitten [W] has shown that prime knots with isomorphic groups have homeomorphic complements. Hence we have

COROLlaRY 1.1. If two prime knots have isomorphic groups then they are equivalent.

The notion of equivalence of knots can be strengthened by saying that $K$ and $K^{\prime}$ are isotopic if the above homeomorphism $h$ is isotopic to the identity, or equivalently, orientation-preserving. The analog of Theorem 1 holds in this setting too: if two knots have complements which are homeomorphic by an orientation-preserving homeomorphism, then they are isotopic.

Theorem 1 and its orientation-preserving version are easy consequences of the following theorem concerning Dehn surgery. $S^{3}$.

THEOREM 2. Nontrivial Dehn surgery on a nontrivial knot never yields

The arguments used to prove Theorem 2 also lead to restrictions on when Dehn surgery on a knot yields a reducible manifold. (It is conjectured that this happens only with torus knots and cable knots.)

THEOREM 3. If a 3-manifold obtained by Dehn surgery on a nontrivial knot is reducible then it has a lens space as a connected summand.

COROLlary 3.1. Any homology 3-sphere obtained by Dehn surgery on a knot is irreducible.

Theorem 3 also gives a new proof of the following result of Gabai [Ga], which includes the Property $R$ Conjecture.

Received by the editors June 8, 1988.

1980 Mathematics Subject Classification (1985 Revision). Primary 57M25. 
COROLlaRY 3.2 (GABAI). Any 3-manifold obtained by a 0-framed surgery on a nontrivial knot is irreducible.

SKeTCH PROOF OF TheOReM 2. Let $K$ be a knot in $S^{3}$, with tubular neighborhood $N(K)$, and let $X=S^{3}-\dot{N}(K)$ be the exterior of $K$. Let $\rho$ be a slope on $\partial X$, that is, the unoriented isotopy class of an essential simple loop on $\partial X$. Let $K(\rho)$ denote the closed 3-manifold obtained by $\rho$-Dehn surgery on $K$, in other words, the result of attaching a solid torus $V$ to $X$ so that $\rho$ bounds a disk in $V$. Let $\mu$ be the slope of a meridian of $K$. Then the trivial Dehn surgery yields $K(\mu) \cong S^{3}$. Let $\mu^{\prime}$ be another slope on $\partial X$, having minimal geometric intersection number $n \geq 1$ with $\mu$.

The starting point of the proof of Theorem 2 is the following proposition. David Gabai has independently proved this proposition.

PRoposition 1. If $K\left(\mu^{\prime}\right)$ is homeomorphic to $S^{3}$, then there exist planar surfaces $P, P^{\prime}$ properly embedded in $X$ such that

(i) $\partial P\left(\partial P^{\prime}\right)$ consists of parallel copies of $\mu$ (resp. $\left.\mu^{\prime}\right)$;

(ii) $P$ and $P^{\prime}$ are in mutual general position, and each component of $\partial P$ intersects each component of $\partial P^{\prime}$ in $n$ points;

(iii) no arc of $P \cap P^{\prime}$ is boundary-parallel in either $P$ or $P^{\prime}$.

The construction of $P$ and $P^{\prime}$ is based on [Ga, $\left.\S 4(\mathrm{~A})\right] . P$ is the intersection with $X$ of a suitable level 2-sphere in $S^{3}$ for a height function $h$ with respect to which $K$ is in thin presentation [Ga, p. 491]. Since $K\left(\mu^{\prime}\right)$ is homeomorphic to $S^{3}$, the core of the attached solid torus $V$ is a knot $K^{\prime}$, say, and $P^{\prime}$ likewise comes from a level 2-sphere of a height function $h^{\prime}$ with respect to which $K^{\prime}$ is in thin presentation. Given $P^{\prime}$, the argument in [Ga, $\S 4(\mathrm{~A})]$ produces $P$ such that (i) and (ii) hold and no arc of $P \cap P^{\prime}$ is boundary-parallel in $P^{\prime}$. Similarly, given $P$, one can find $P^{\prime}$ satisfying (i) and (ii) such that no arc on $P \cap P^{\prime}$ is boundary-parallel in $P$. The additional content of Proposition 1 is that we can find $P$ and $P^{\prime}$ so that these conditions hold simultaneously.

To do this, we pick a 1-parameter family of level 2-spheres of $h$ in $K(\mu)$ between an adjacent local maximum and local minimum of $K$. This family becomes a 1-parameter family $\{P(\lambda)\}$ of punctured 2-spheres in $X$. We put the family $\{P(\lambda)\}$ in general position with respect to the height function $h^{\prime} \mid X$. This means that for all but finitely many $\lambda, h^{\prime} \mid P(\lambda)$ is a Morse function, and each $P(\lambda)$ such that $h^{\prime} \mid P(\lambda)$ is not Morse has a single singularity corresponding to a birth, death or an exchange of tangencies.

Assume for a contradiction that Proposition 1 is false. The argument in [Ga, $\S 4(\mathrm{~A})$ ], using the thin presentation of $K^{\prime}$, allows one to associate to each $P(\lambda)$ such that $h^{\prime} \mid P(\lambda)$ is Morse, a punctured level 2-sphere of $h^{\prime}$, say $P_{\lambda}^{\prime}$, which intersects $P(\lambda)$ transversely and is such that $P(\lambda) \cap P_{\lambda}^{\prime}$ contains an arc which is boundary-parallel in $P_{\lambda}^{\prime}$. If the corresponding arc in $\partial P_{\lambda}^{\prime}$ lies above (below) $P(\lambda)$ in $K(\mu)$ then $P(\lambda)$ is called low (high, respectively), as in [Ga, $\S 4(\mathrm{~A})]$. One observes that as $\lambda$ increases, $P(\lambda)$ starts off high and ends up low. By the thinness of $K$ under $h$, a change from high to low in 
$\{P(\lambda)\}$ can only occur at a $\lambda_{0}$ such that $h^{\prime} \mid P\left(\lambda_{0}\right)$ is not Morse. One analyses what happens at $P\left(\lambda_{0}\right)$ using the special way in which $P_{\lambda}^{\prime}$ is constructed for $P(\lambda)$, and eventually arrives at a contradiction to the thinness of $K$ under $h$.

To prove Theorem 2 we now carry out a combinatorial analysis of the intersection of the planar surfaces $P$ and $P^{\prime}$, ultimately deriving a contradiction. More precisely, we cyclically number the boundary components of $P\left(P^{\prime}\right)$ in the order they occur on $\partial X$, and label the endpoints of the arcs of $P \cap P^{\prime}$ in $P\left(P^{\prime}\right)$ with the corresponding boundary component of $P^{\prime}(P)$. Assigning (arbitrary) orientations to $P$ and $P^{\prime}$ allows us to refer to + and - boundary components of $P$ and $P^{\prime}$, according to the direction of the induced orientation of a boundary component as it lies on $\partial X$. Then for any arc $\alpha$ of $P \cap P^{\prime}$, the boundary components of $P$ joined by $\alpha$ on $P$ have the same sign if and only if the boundary components of $P^{\prime}$ joined by $\alpha$ on $P^{\prime}$ have opposite sign. Capping off the boundary components of $P\left(P^{\prime}\right)$ with disks, we regard these disks as forming the 'fat' vertices of a graph $\Gamma\left(\Gamma^{\prime}\right)$ in $S^{2}$, the edges of $\Gamma\left(\Gamma^{\prime}\right)$ corresponding to the arcs of $P \cap P^{\prime}$ in $P\left(P^{\prime}\right)$. We thus obtain two labeled graphs in $S^{2}$, whose edges are in one-one correspondence, such that the labeling satisfies the sign condition noted above, and such that neither graph contains a trivial loop (by condition (iii) of Proposition 1).

A (disk) face of $\Gamma^{\prime}$ corresponds to a subdisk of $P^{\prime}$ which we may regard as lying in $K(\mu)$ with its boundary contained in $P \cup \partial N(K)$. Similarly, the faces of $\Gamma$ may be regarded as lying in $K\left(\mu^{\prime}\right)$. This allows us to infer topological properties of $K(\mu)\left(K\left(\mu^{\prime}\right)\right)$ from graph-theoretic properties of $\Gamma^{\prime}(\Gamma)$.

For $n \geq 2$, this program is already carried out in [CGLS, $\S 2.5$ and 2.6; see Proposition 2.5.6]. There it is shown that given a pair of graphs $\Gamma, \Gamma^{\prime}$ as above, one of them, say $\Gamma$, contains a special kind of face (a Scharlemann cycle), which implies that $K\left(\mu^{\prime}\right)$ contains a punctured lens space.

We therefore focus on the case $n=1$, where the above assertion is false and more delicate graph-theoretic arguments are needed. To describe the result, note that a disk face of $\Gamma^{\prime}$ (say) corresponds to a disk $E$ in $P^{\prime}$ whose boundary $\partial E$ can be expressed as a sequence of arcs $a_{1}, b_{1}, \ldots, a_{k}, b_{k}$, where $a_{i}$ lies in $P \cap P^{\prime}$ and $b_{i}$ lies in $\partial N(K), 1 \leq i \leq k$. If the boundary components of $P$ are labeled $1, \ldots, p$ (in order on $\partial N(K)$ ), then each $b_{i}$ joins some consecutive pair of boundary components $j_{i}, j_{i}+1$. Orienting $\partial E$, we regard $b_{i}$ as representing $\left(j_{i}, j_{i}+1\right)$ or $-\left(j_{i}, j_{i}+1\right)$ according as $b_{i}$ runs from $j_{i}$ to $j_{i}+1$ or vice versa. In this way, $\partial E$ represents an element $\alpha(E)=\left(\alpha_{1}(E), \ldots, \alpha_{p}(E)\right)$ of the free abelian group on the set of consecutive label-pairs $(1,2),(2,3), \ldots,(p-1, p),(p, 1)$. We say that $\Gamma^{\prime}$ represents all types if there exists a collection $\mathscr{E}$ of disk faces of $\Gamma^{\prime}$ such that

(1) for each $E \in \mathscr{E}$ and for each label-pair $(j, j+1)$, all occurrences of $(j, j+1)$ in $\partial E$ have the same sign; 
(2) for each sequence $\left(\varepsilon_{1}, \ldots, \varepsilon_{p}\right)$, where $\varepsilon_{i}= \pm, 1 \leq i \leq p$, there exists $E \in \mathscr{E}$ and $\eta= \pm$ such that

$$
\operatorname{sign} \alpha_{i}(E)=\eta \varepsilon_{i} \text { for all } i \text { such that } \alpha_{i}(E) \neq 0 \text {. }
$$

(Clearly (1) and (2) are independent of the orientation of $\partial E$.)

We remark that if $\Gamma^{\prime}$ contains a Scharlemann cycle $E$, then taking $\mathscr{E}=$ $\{E\}$ shows that $\Gamma^{\prime}$ represents all types.

We prove the following result.

Proposition 2. Let $\Gamma, \Gamma^{\prime}$ be a pair of graphs as described above. Either $\Gamma$ contains a Scharlemann cycle or $\Gamma^{\prime}$ represents all types.

The final step in the proof of Theorem 2 is supplied by the following proposition (in which we do not assume that $K\left(\mu^{\prime}\right)$ is $S^{3}$ ).

Proposition 3. Suppose that $X$ contains properly embedded planar surfaces $P, P^{\prime}$ satisfying conditions (i), (ii) and (iii) of Proposition 1, where $P$ is the intersection with $X$ of a level 2-sphere in a thin presentation of $K$. Let $\Gamma, \Gamma^{\prime}$ be the associated graphs. Then $\Gamma^{\prime}$ does not represent all types.

Proposition 3 is proved by showing that under the given hypotheses, a collection $\mathscr{E}$ of faces of $\Gamma^{\prime}$ representing all types would ultimately lead to the existence of a punctured lens space in $K(\mu) \cong S^{3}$, which is absurd.

Propositions 2 and 3 imply that $K\left(\mu^{\prime}\right)$ contains a punctured lens space. Since, in the context of Theorem $2, K\left(\mu^{\prime}\right)$ is also homeomorphic to $S^{3}$, this contradiction completes the proof.

SKeTCH PROOF OF THEOREM 3. Let $\mu^{\prime}$ be a slope on $\partial X$ such that $K\left(\mu^{\prime}\right)$ is reducible. Then there exists a properly embedded, incompressible (and non-boundary-parallel) planar surface $P^{\prime}$ in $X$ whose boundary components have slope $\mu^{\prime}$. By [Ga, $\left.\S 4(\mathrm{~A})\right]$ there is a planar surface $P$ in $X$, coming from a level sphere in a thin presentation of $K$, such that $P$ and $P^{\prime}$ satisfy conditions (i), (ii), and (iii) of Proposition 1 . Exactly as in the proof of Theorem 2, Propositions 2 and 3 now show that $K\left(\mu^{\prime}\right)$ contains a punctured lens space.

We understand that David Gabai and Will Kazez, using a similar approach, have independently obtained some partial results on Theorem 2.

\section{REFERENCES}

[CGLS] M. Culler, C. McA. Gordon, J. Luecke, and P. B. Shalen, Dehn surgery on knots, Ann. of Math. (2) 125 (1987), 237-300.

[Ga] D. Gabai, Foliations and the topology of 3-manifolds. III, J. Differential Geom. 26 (1987), 479-536.

[T] H. Tietze, Über die topologischen Invarianten mehrdimensionaler Mannigfaltigkeiten, Monatsh. Math. Phys. 19 (1908), 1-118.

[W] W. Whitten, Knot complements and groups, Topology 26 (1987), 41-44. 
Department of Mathematics, University of Texas at Austin, Austin, Texas 78712

Department of Mathematics, Courant Institute, 251 Mercer Street, New York, NEW YORK 10012

CURrent address (J. Luecke): Department of Mathematics, University of TeXas at Austin, Austin, Texas 78712 
